\title{
THE GLUON CONTENT OF THE PION FROM HIGH-p, DIRECT PHOTON PRODUCTION
}

\author{
P. AURENCHE \\ Fermi National Accelerator Laboratory, Batavia, IL 60439, USA \\ and LAPP, B.P. 110, F-74941 Annecy-le-Vieux Cedex, France \\ R. BAIER \\ Fakultät für Physik, Universität Bielefeld, D-4800 Bielefeld I, FRG \\ M. FONTANNAZ \\ LPTHE, Bâtiment 211, Université de Paris XI, F-91405 Orsay Cedex, France
}

M.N. KIENZLE-FOCACCI

DPNC, Universitè de Genève, CH-1211 Geneva 4, Switzerland

and

\author{
M. WERLEN \\ IPN, Université de Lausanne, BSP Dorigny, CH-1015 Lausanne, Switzerland
}

Received 19 September 1989

\begin{abstract}
A fit to the large $p_{t}$ direct photon cross-sections for the reactions $\pi^{ \pm} p \rightarrow \gamma \mathrm{X}$, using complete beyond leading logarithm QCD expressions, allows the determination of the content of gluons and valence quarks inside the pion and defines the shape of the gluon structure function for $x>0.2$. The QCD scale parameter $A_{\overline{\mathrm{MS}}}$ is in good agreement with the most precise deep inelastic scattering determinations.
\end{abstract}

Direct photon production by $\pi^{+}$and $\pi^{-}$beams incident on protons is well described [1] by QCD calculations complete up to $\mathrm{O}\left(\alpha_{\mathrm{s}}^{2}\right)$ [2]. Up to now, the comparison between theory and experimental data was done using the values of the QCD scale parameter $A$ and the structure functions determined by other experiments. In particular the set $1(A=0.200 \mathrm{GeV})$ of the leading order parametrization of Duke and Owens for nucleons [3] and pions [4] was found to give satisfactory agreement within the systematic errors of the experiment. However, while the valence quark distribution is well determined by deep inelastic scattering (DIS) for the proton and by Drell-Yan experiments (D-Y) for the pion, the gluon structure functions are rather indirectly fixed by DIS and $\mathrm{J} / \psi$ production. There is also a correlation between the values of parameters which enter the theoretical predictions, for example between the structure functions and the value of $\Lambda$. In direct photon production, the $\mathrm{pp} \rightarrow \gamma \mathrm{X}$ and $\pi^{+} \mathrm{p} \rightarrow \gamma \mathrm{X}$ cross sections are dominated by qg scattering, thus directly sensitive to the gluon distributions while the difference of cross sections $\sigma\left(\pi^{-} p\right)-\sigma\left(\pi^{+} p\right)$ is dominated by $q \bar{q}$ annihilation, thus providing a sensitive determination of $A$. The $p p \rightarrow \gamma \mathrm{X}$ data of the WA70 experiment [1] were already used [5], together with the DIS data of the BCDMS Collaboration [6], to determine beyond leading logarithms the value of $A$ and the gluon structure function of the proton. In this paper we analyze in a similar way the $\pi^{-} p$ and $\pi^{+} p$ WA70 data [1], integrated with respect to the variable $x_{F}$ $\left(\left|x_{F}\right|<0.45\right)$, to determine the gluon structure func- 
tion of the pion and its relative weight to the valence distribution. The data of the NA24 experiment [7], well compatible with WA70 results, are also separately fitted.

The cross sections are calculated [2] beyond leading order, up to $\mathrm{O}\left(\alpha_{\mathrm{s}}^{2} \alpha\right)$, in the $\overline{\mathrm{MS}}$ factorization and renormalization schemes and four flavors are used throughout the calculation. The Principle of Minimal Sensitivity [8] ${ }^{\# 1}$ determines the optimum values of the factorization scale $M$ (which appears in the distribution functions) and of the renormalization scale $\mu$ (which is the argument of the strong coupling constant). These optimum values are taken to represent the stability domain of the cross-sections, i.e. the domain where the cross-sections are not influenced by rather large changes of the (unphysical) scales $M$ and $\mu$. The structure functions necessary to describe the experimental cross sections are the distributions of the valence quarks, the gluon and the sea inside the pion and the proton. They are parametrized at $Q_{0}^{2}=2 \mathrm{GeV}^{2}$ and evolved to different $Q^{2}$ with the second order Altarelli-Parisi equations using the updated code from ref. [10]. The pion structure functions are parametrized at $Q_{0}^{2}=2 \mathrm{GeV}^{2}$ by the forms

$$
\begin{aligned}
& x V(x)=N_{\mathrm{v}}(\alpha, \beta) x^{\alpha}(1-x)^{\beta}, \quad \beta=0.85, \\
& x S(x)=2 x[\bar{u}(x)+\bar{d}(x)+\bar{s}(x)]=N_{\mathrm{s}}(1-x)^{\delta}, \\
& \delta=7.5, \quad\langle x S(x)\rangle=0.14, \\
& x G(x)=N_{\mathrm{g}}(1-x)^{\eta},
\end{aligned}
$$

respectively for the valence, total sea and gluon distributions, assuming the same distribution for valence quark and antiquark and SU(3) symmetry for the sea. The relative contribution of the three components is constrained by the momentum sum rule $\int \mathrm{d} x[2 x V(x)+x S(x)+x G(x)]=1$.

The parameter $\beta$ and the sea contribution are taken as external inputs from D-Y analyses which determine the structure functions at $\left\langle Q^{2}\right\rangle=30 \mathrm{GeV}^{2}$ and the value of these parameters are therefore extrapolated to $Q_{0}^{2}=2 \mathrm{GeV}^{2}$. A number of $\mathrm{D}-\mathrm{Y}$ experiments [11-14] with $\pi^{-}$beams incident on heavy targets

\#1 For details on the use of the optimization approach in real photon processes see ref. [9]. have studied the valence quark distribution. However, fits to the data are sensitive to the assumed value of $A$, to the normalization $K$-factor and to the nucleon valence parameters, which may be taken from various DIS experiments. A consistent analysis of all the data is missing. The difficulty to have a unique value for $x V(x)$ is illustrated in fig. 1. Only the parameter $\beta$ has therefore been fixed to a value consistent with D-Y experiments inside their quoted errors, while the valence contribution $2\langle x V(x)\rangle=2 \alpha$ / $(1+\beta+\alpha)$ is a free parameter in the fit. The sea distribution was determined only by NA3 [11] and it has been fixed to its value. For the proton we use the set of structure functions determined by the combined analysis of BCDMS and WA70 data [5].

First we have performed a least squares fit between the theoretical predictions and the 13 data points of the WA70 experiment $\left(p_{\mathrm{t}}>4.25 \mathrm{GeV} / c\right)$ using only statistical errors, with $\Lambda$ (in the expression for $\alpha_{\mathrm{s}}$ ), $2\langle x V(x)\rangle$ and $\eta$ as free parameters. In the structure function evolution, for both pion and proton, $A$ is fixed to $0.231 \mathrm{GeV}$, the value obtained by the combined BCDMS and WA70 analysis [5]. The results of the fit are listed in table 1; the data are well repro-

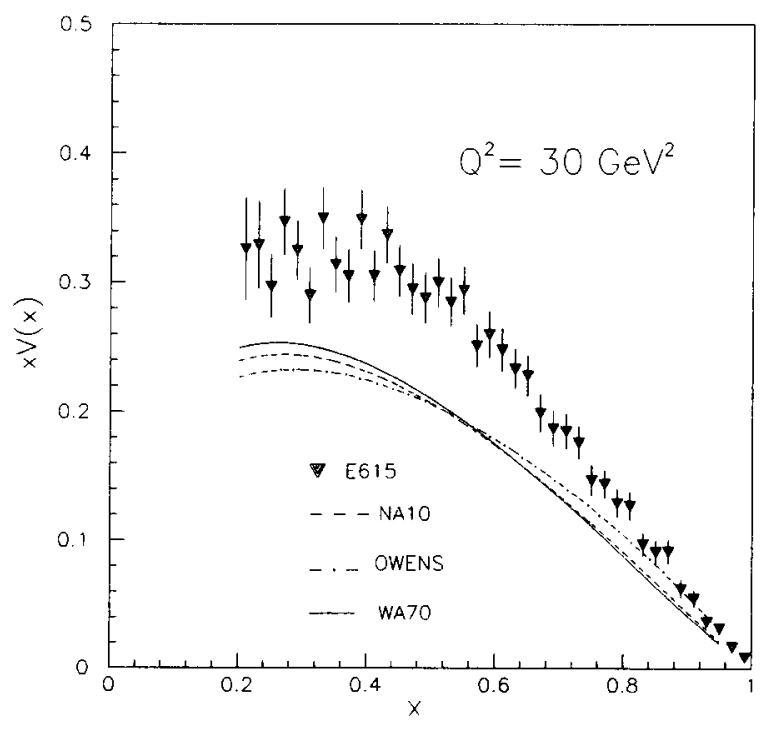

Fig. 1. A compilation of the valence structure function of the pion from Drell-Yan data [11-14] and from the Owens [4] set 1 parametrization for $Q^{2}=30 \mathrm{GeV}^{2}$. The NA3 and E537 results (not shown ) are compatible with the fit from NA 10. Superimposed as a continuous line is the result of the fit to direct photon data from WA70. 
Table 1

Best fit to direct photon data.

\begin{tabular}{|c|c|c|c|c|}
\hline Collaboration & $A(\mathrm{GeV})$ & $\eta$ & $2\langle x V(x)\rangle$ & $\chi^{2} /$ dof \\
\hline \multicolumn{5}{|c|}{ scales defined by Principle of Minimal Sensitivity } \\
\hline WA70 & $0.229 \pm 0.043$ & $1.86 \pm 0.42$ & $0.415 \pm 0.042$ & $9.8 / 10$ \\
\hline WA70 & $\begin{array}{l}0.231 \text { fixed } \\
\text { systematic error }\end{array}$ & $\begin{array}{r}1.94 \pm 0.20 \\
+0.39 \\
-0.17\end{array}$ & $\begin{array}{r}0.413 \pm 0.020 \\
+0.052 \\
-0.030\end{array}$ & $9.9 / 11$ \\
\hline NA24 & 0.231 fixed & $1.82 \pm 0.37$ & $0.338 \pm 0.046$ & $8.6 / 7$ \\
\hline \multicolumn{5}{|c|}{ scales defined by $M=\mu=p_{t} / 2$} \\
\hline WA70 & 0.231 fixed & $1.48 \pm 0.21$ & $0.475 \pm 0.021$ & $17 / 11$ \\
\hline \multicolumn{5}{|c|}{ scales defined by $M=\mu=p_{\imath}$} \\
\hline
\end{tabular}

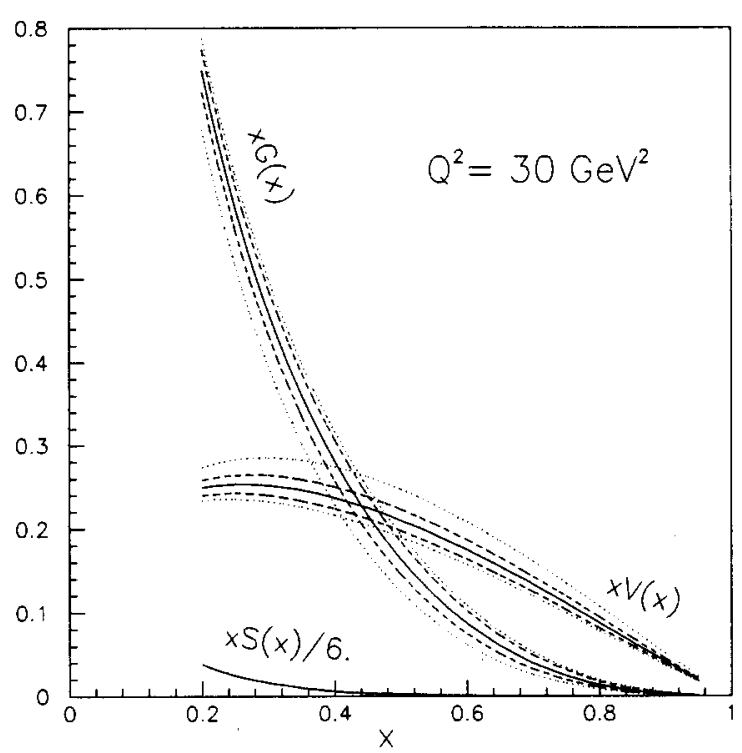

Fig. 2. The pion structure functions obtained with direct photon data for $Q^{2}=30 \mathrm{GeV}^{2}$. The continuous line is the central value of the fit, the dashed line indicates the statistical errors and the dotted one the systematic errors of the WA70 experiment. The sea distribution is taken from NA3 [11].

duced by the theory with a value of $A$ completely consistent with the one determined by the previous analysis [5]. There are strong correlations between the three parameters, the correlation coefficient between $A$ and $\eta$ is 0.89 , between $A$ and $2\langle x V(x)\rangle$ is -0.88 , between $2\langle x V(x)\rangle$ and $\eta$ is -0.62 . Since in the pre- vious analysis [5] the precision of the $A$ parameter is mainly due to the high statistics DIS data, this parameter is finally fixed to its value of $0.231 \mathrm{GeV}$, to improve the accuracy of the pion structure function parameters. The results of this fit are also listed in table 1 , the central value of the pion parameters is unaffected by the constraint on $A$, but the errors are considerably reduced. The correlation coefficient between $2\langle x V(x)\rangle$ and $\eta$ is 0.77 . The systematic errors due to experimental uncertainties are estimated by repeating the same fit with statistical and systematic errors added in quadrature. The same fit to the NA24 data ( 9 points with $p_{\mathrm{t}}$ above $3.5 \mathrm{GeV} / c$ [7]) gives consistent results (table 1 ).

The shapes of the pion structure functions thus obtained are drawn in fig. 2 for $Q^{2}=30 \mathrm{GeV}^{2}$ together with the statistical and systematic uncertainty due to the WA70 data. The valence structure function is compared to D-Y determinations in fig. 1 (continuous line). The present results are similar to most of the D-Y determinations and to the Owens parametrization, but $\approx 20 \%$ lower than the recent determination by the experiment E615. A non-perturbative QCD lattice calculation [15] finds a value of $0.49 \pm 0.08$ for the first moment of the valence quarks in the pion at $Q^{2}=49 \mathrm{GeV}^{2}$; our parametrization gives $0.338 \pm 0.017$ (stat.) [ $+0.043,-0.024$ (syst.) ] when evolved to this $Q^{2}$. Previously the gluon structure function of the pion was mainly determined from $\mathrm{J} / \psi$ and $\Upsilon$ production [16,17] on heavy nuclei, assuming that these resonances are produced in $\pi^{-} \mathrm{N}$ 
reactions via gluon-gluon fusion using a leading order QCD formalism. In fig. 3 the direct photon result is compared with determinations from $J / \psi[16,4]$ at $Q^{2}=10 \mathrm{GeV}^{2}$; the gluon distribution of the present analysis is below the previous determinations. For the $\Upsilon$ production, the NA10 experiment [17] finds the same value of $\eta$ and keeps the same average value of $G(x)$ as for $\mathrm{J} / \psi$ production; this result is rather difficult to understand if the gluon structure function is evolved to a $Q^{2}$ corresponding to the $\Upsilon$ mass. We recall however that our analysis of the gluon distribution uses a next-to-leading logarithms formalism whereas the previous determinations, from heavy resonance production $[4,16,17]$, were based on a leading logarithm approach and one should therefore be very cautious before drawing conclusions form the comparison.

We have repeated the fit to the WA70 data with a fixed choice of factorization and renormalization scales. Imposing $M=\mu=p_{\mathrm{t}} / 2$ the quality of the fit worsens (table 1 ), but the parameters $\eta$ and $2\langle x V(x)\rangle$ change only by two and three standard deviations respectively. This choice of scales gives therefore a fair representation for the stability region of the theoretical cross-sections in the kinematical

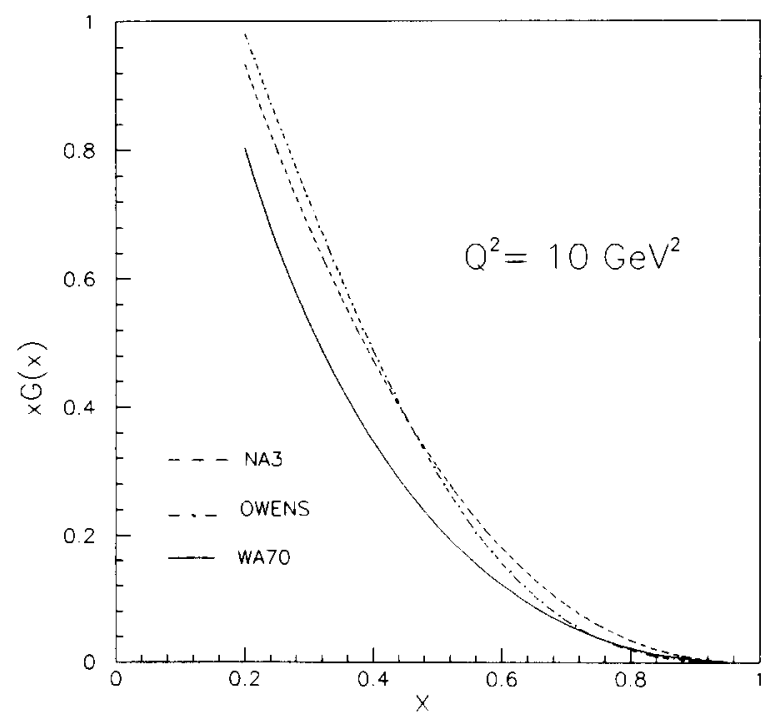

Fig. 3. Gluon structure function of the pion at $Q^{2}=10 \mathrm{GeV}^{2}$ from the WA70 experiment (continuous line) compared to the parametrization of Owens [4] set 1 (dash-dotted line) and to the fit of $J / \psi$ data of NA3 [16] (dashed line). range explored by the data under investigation. In contrast a conventional choice of scales $M=\mu=p_{t}$, does not allow to reproduce the data with the $A$ parameter fixed to $0.231 \mathrm{GeV}$. In this case it is necessary to leave $A$ free to reach a higher value: $A=0.688$ $\mathrm{GeV}$ (table 1). A similar pattern has also been obtained in fitting the $\mathrm{pp} \rightarrow \gamma \mathrm{X}$ reaction [5]. Despite the unreasonable value of $A$, the other parameters change by less than one standard deviation.

The sensitivity of this fit to a variation of the parameters fixed by other analysis is difficult to quantify since all parameters are highly correlated. An indication of the sensitivity is given in table 2 where the variation of $\eta$ and $2\langle x V(x)\rangle$ is obtained by repeating the fit to the WA70 data for the two extreme values each fixed parameter can assume. The parameter $\beta$ of the pion valence structure function, varied between the different estimates of the $\mathrm{D}-\mathrm{Y}$ experiments, gives a small effect. When the sea parameters are varied inside the uncertainties given by NA3, the shape of the gluon distribution appears rather sensitive to the sea normalization, while no effect is observable on the average valence distribution. To estimate the errors induced by the proton structure functions determination we take the proton gluon shape parameter $\eta_{\mathrm{p}}$ and the QCD parameter $A$ to the limit of their systematic errors and we find that the effect on the pion structure functions is of the same order as the experimental systematic errors (table 2 ).

In conclusion the analysis of high $p_{\mathrm{t}}$ direct photon data produced by $280 \mathrm{GeV} / c \pi^{+}$and $\pi^{-}$incident on hydrogen, in the framework of a QCD calculation complete up to the order $\alpha_{s}^{2} \alpha$ with the choice of scales defined by the Principle of Minimal Sensitivity, gives a scale parameter of QCD $A$, in the $\overline{\mathrm{MS}}$ scheme, consistent with the most precise deep inelastic scattering

Table 2

Sensitivity to variations of the fixed parameters.

\begin{tabular}{llll}
\hline Parameter & Variation & $\delta \eta$ & $\delta(2\langle x V(x)\rangle)$ \\
\hline$\beta=0.85$ & \pm 0.15 & $\mp 0.04$ & \pm 0.005 \\
$\delta=7.5$ & +2.2 & -0.03 & \\
& -2.2 & +0.07 & \\
$\langle x S(x)\rangle=0.14$ & \pm 0.05 & $\mp 0.25$ & \\
$\eta_{\mathrm{p}}=4.0$ & +0.8 & -0.23 & +0.001 \\
& -0.6 & +0.27 & -0.001 \\
$A=0.231$ & +0.05 & +0.37 & -0.041 \\
& -0.05 & -0.34 & +0.054 \\
\hline
\end{tabular}


determinations [5]. The average momentum of the pion valence at $Q^{2}=2 \mathrm{GeV}^{2}$ is found to be

$$
\begin{aligned}
2 & \langle x V(x)\rangle \\
& =0.413 \pm 0.020 \text { (stat.) }[+0.052,-0.030 \text { (syst.) }],
\end{aligned}
$$

compatible with most of Drell-Yan experiments [1113], but lower than expected by the recent E615 analysis [14]. The gluon structure function of the pion at $Q^{2}=2 \mathrm{GeV}^{2}$ is found to be

$$
\begin{aligned}
& x G(x)=1.3(1-x)^{\eta}, \\
& \eta=1.94 \pm 0.20(\text { stat. })[+0.39,-0.17 \text { (syst. })] .
\end{aligned}
$$

If the choice of factorization and renormalization scales is left open, the theory predictions may vary considerably: high values of $\mu^{2}$ and $M^{2}$ must be compensated by a large value of $A(A=0.688 \mathrm{GeV}$ for the conventional choice $M=\mu=p_{1}$ ), but the structure functions parameters remain inside the statistical and systematic errors of the experiments.

We are grateful to K. Freudenreich for helpful discussions on the Drell-Yan experiments and to the whole WA70 Collaboration for encouragement.

\section{References}

[1] WA70 Collab., M. Bonesini et al., Z. Phys. C 37 (1988) 535; C 38 (1988) 371.
[2] P. Aurenche, R. Baier, A. Douiri, M. Fontannaz and D. Schiff, Phys. Lett. B 140 (1984) 87; Nucl. Phys. B 297 (1988) 661 .

[3] D.W. Duke and J.F. Owens, Phys. Rev. D 30 (1984) 49.

[4] J.F. Owens, Phys. Rev. D 30 (1984) 943.

[5] P. Aurenche, R. Baier, M. Fontannaz, J.F. Owens and M Werlen, Phys. Rev. D 39 (1989) 3275.

[6] BCDMS Collab., A.C. Benvenuti et al., Phys. Lett. B 223 (1989) 485, 490.

[7] NA24 Collab., C. de Marzo et al., Phys. Rev. D 36 (1987) 8.

[8] P.M. Stevenson, Phys. Rev. D 23 ( 1981 ) 2916; P. Stevenson and H.D. Politzer, Nucl. Phys. B 277 (1986) 758.

[9] P. Aurenche, R. Baier, M. Fontannaz and D. Schiff, Nucl. Phys. B 286 ( 1987) 509.

[10] A. Devoto, D.W. Duke, J.F. Owens and R.G. Roberts, Phys. Rev. D 27 ( 1983 ) 508.

[11] NA3 Collab., J. Badier et al., Z. Phys. C 18 (1983) 281.

[12] NA10 Collab., B. Betev et al., Z. Phys. C 28 (1985) 15;

K. Freudenreich, Habilitation Dissertation, ETHZ, Zurich (1989).

[13] E537 Collab., E. Anassontzis et al., Phys. Rev. D 38 (1988) 1377.

[14] E615 Collab., J.S. Conway et al., Phys. Rev. D 39 (1989) 92.

[15] G. Martinelli and C.T. Sachrajda, Phys. Lett. B 196 (1987) 184.

[16] NA3 Collab., J. Badier et al., Z. Phys. C 20 (1983) 101; WA1l Collab., J.G. McEwen et al., Phys. Lett. B 121 (1983) 198.

[17] NA 10 Collab., M. Grossmann-Handschin et al., Phys. Lett. B 179 (1986) 170. 